\title{
Thermal Diffusion and Diffusion Thermo Effects on the Viscous Fluid Flow with Heat and Mass Transfer through Porous Medium over a Shrinking Sheet
}

\author{
Nabil Eldabe ${ }^{1}$ and Mahmoud Abu Zeid ${ }^{2}$ \\ ${ }^{1}$ Department of Mathematics, Faculty of Education, Ain Shams University, Roxy, Heliopolis, Cairo, Egypt \\ ${ }^{2}$ Basic Science Department, Higher Technological Institute, 10th of Ramadan City, Egypt
}

Correspondence should be addressed to Mahmoud Abu Zeid; abuzeid71@yahoo.com

Received 9 February 2013; Revised 16 April 2013; Accepted 18 April 2013

Academic Editor: Magdy A. Ezzat

Copyright (C) 2013 N. Eldabe and M. Abu Zeid. This is an open access article distributed under the Creative Commons Attribution License, which permits unrestricted use, distribution, and reproduction in any medium, provided the original work is properly cited.

\begin{abstract}
The motion of the viscous, incompressible fluid through a porous medium with heat and mass transfer over a shrinking sheet is investigated. The cross-diffusion effect between temperature and concentration is considered. This phenomenon is modulated mathematically by a set of partial differential equations which govern the continuity, momentum, heat, and mass. These equations are transformed to a set of ordinary differential equations by using similarity solutions. The analytical solutions of these equations are obtained. The velocity, temperature, and concentration of the fluid as well as the heat and mass transfer with shear stress at the sheet are obtained as a function of the physical parameters of the problem. The effects of Prandtl number, mass transfer parameter, the wall shrinking parameter, the permeability parameter, and Dufour and Soret numbers on temperature and concentration are studied. Also, the effects of mass transfer parameter, permeability parameter, and shrinking strength on the velocity and shear stress are discussed. These effects are illustrated graphically through a set of figures.
\end{abstract}

\section{Introduction}

The study of two-dimensional boundary layer flow of an incompressible fluid over a stretching or shrinking surface has assumed significance in recent years because of its extensive applications in engineering disciplines. Some applications are glass fiber production, hot rolling and wire drawing, the aerodynamic extrusion of plastic sheets, paper production, glass blowing, and drawing plastic films. The flow over a continuously stretching surface with a constant speed was initiated by Sakiadis $[1,2]$ and had been extended to the different physical situations [3-5]. The effect of large suction on the MHD forced and free convection flow past a vertical porous plate was studied by Ferdows et al. [6]. Lin et al. [7] studied the flow characteristics of an electrically conducting second-order fluid over a stretching sheet with suction velocity in the presence of a transverse magnetic field. P. S. Gupta and A. S. Gupta [8] studied the heat and mass transfer in a stretching surface with suction or injection.
Chen and Char [9] studied the effects of variable surface temperature and variable surface heat flux on the heat transfer characteristics of a linearly stretching sheet. The MHD effect on a vertical stretching surface with suction and blowing was studied by Gorla et al. [10]. Yao et al. [11] investigated heat transfer problem with a convective boundary condition for a viscous and incompressible fluid over a permeable (with mass flux) stretching/shrinking sheet in a quiescent fluid. Fang et al. [12] investigate the behavior of the steady boundary layer flow and heat transfer of a viscous and incompressible fluid towards a permeable stretching/shrinking sheet in a quiescent fluid.

Recently, the flow over a shrinking sheet has attracted the attention of many researchers. Fang [13], Fang et al. [14], Fang and Zhang [15], and Fang et al. [16] studied problems of boundary layer flow over shrinking sheets with mass transfer. The flow induced by a shrinking sheet shows physical phenomena distinct from the forward stretching flow. A steady flow over a shrinking sheet is not possible because 


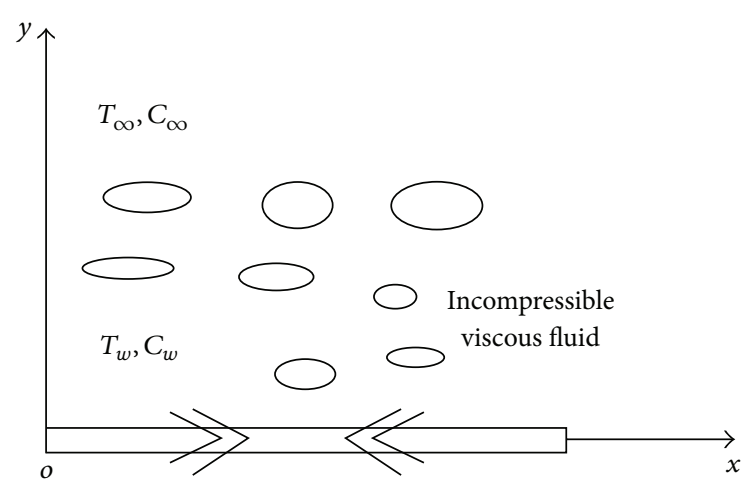

FIgURE 1: The physical configuration of the problem.

the generated vorticity is not confined within the boundary layer. So, to overcome this difficulty the flow needs a certain amount of external opposite force at the sheet. The most suitable external force is the suction at the sheet. Miklavčič and Wang [17] investigated the shrinking flow where velocity of boundary layer moves toward a fixed point. They found an exact solution of the Navier-Stokes equations and reported that an adequate suction is necessary to maintain the flow over a shrinking sheet. This phenomenon can be found, for example, on a rising and shrinking balloon [18].

The heat and mass transfer that simultaneously affecting each other will cause the cross-diffusion effect. The heat transfer caused by concentration gradient is called the diffusionthermo or Dufour effect. On the other hand mass transfer caused by temperature gradient is called Soret or thermaldiffusion effect. Alam and Rahman [19] investigated the Dufour and Soret effects on mixed convection flow past a vertical porous flat plate with variable suction. El-Arabawy [20] investigated the heat and mass transfer by natural convection from vertical surface embedded in a fluid-saturated porous media considering Soret and Dufour effects with variable surface temperature and constant concentration.

Motivated by the above-mentioned investigations, we investigate in this present paper the behavior of flow of a viscous and incompressible fluid through a porous medium over a continuously shrinking sheet with heat and mass transfer in a stationary fluid. The solutions for the momentum, heat, and mass transfer equations are solved analytically, and the solutions are presented in terms of algebraically decaying function. The effects of various physical parameters like the Prandtl number, the mass transfer parameter, the wall shrinking parameter, the permeability parameter, Dufour number, and Soret number on the temperature and concentration profiles are obtained. Also, the effect of mass suction, permeability of the porous medium, and shrinking strength on the velocity and shear stress are studied.

\section{Basic Equations and Exact Solutions}

Consider the steady two-dimensional flow of a viscous and incompressible fluid through a porous medium over a continuously shrinking sheet with heat and mass transfer in a stationary fluid. It is assumed that the velocity of the shrinking sheet is $u_{w}(x)=-b x$, where $b$ is a real number. It is also assumed that constant mass transfer velocity is $v_{w}$. In the present study, the effective viscosity $\mu_{\text {eff }}$ is considered to be identical to the dynamic viscosity $\mu$. The ambient fluid temperature and concentration are $T_{\infty}$ and $C_{\infty}$, and these at the sheet surface are $T_{w}$ and $C_{w}$. The $x$-axis is along the shrinking surface and $y$-axis is perpendicular to it (see Figure 1).

With these assumptions the governing equations can be written as

$$
\begin{gathered}
\frac{\partial u}{\partial x}+\frac{\partial v}{\partial y}=0, \\
u \frac{\partial u}{\partial x}+v \frac{\partial u}{\partial y}=-\frac{1}{\rho} \frac{\partial P}{\partial x}+v \nabla^{2} u-\frac{v}{k^{\prime}} u \\
u \frac{\partial v}{\partial x}+v \frac{\partial v}{\partial y}=-\frac{1}{\rho} \frac{\partial P}{\partial y}+v \nabla^{2} v-\frac{v}{k^{\prime}} v \\
u \frac{\partial T}{\partial x}+v \frac{\partial T}{\partial y}=\sigma \nabla^{2} T+\frac{D_{m} k_{T}}{c_{s} c_{p}} \nabla^{2} C, \\
u \frac{\partial C}{\partial x}+v \frac{\partial C}{\partial y}=D_{m} \nabla^{2} C+\frac{D_{m} k_{T}}{T_{m}} \nabla^{2} T
\end{gathered}
$$

where $u$ and $v$ are the velocity components along the $x$ and $y$-axes, respectively, $P$ is the pressure, $\rho$ is the density, $v$ is the fluid kinematic viscosity, $k^{\prime}$ is the permeability of porous medium, $\sigma$ is the fluid thermal diffusivity, $D_{m}$ is the coefficient of mass diffusivity, $c_{p}$ is the specific heat at constant pressure, $T_{m}$ is the mean fluid temperature, $k_{T}$ is the thermal diffusion ratio, $c_{s}$ is the concentration susceptibility, $T$ is the fluid temperature, and $C$ is the concentration of the fluid. The appropriate boundary conditions are

$$
\begin{array}{r}
u=u_{w}(x)=-b x, \quad v=v_{w}, \quad T=T_{w}, \quad C=C_{w} \\
\text { at } y=0, \\
u=0, \quad T=T_{\infty}, \quad C=C_{\infty} \quad \text { as } y \longrightarrow \infty .
\end{array}
$$

We assume that (1) to (5) subject to the boundary conditions (6) admit the similarity solutions

$$
\begin{array}{r}
u=a x f^{\prime}(\eta), \quad v=-\sqrt{a v} f(\eta), \\
T-T_{\infty}=\theta(\eta)\left(T_{w}-T_{\infty}\right), \\
C-C_{\infty}=\phi(\eta)\left(C_{w}-C_{\infty}\right), \\
\eta=y \sqrt{\frac{a}{v},}
\end{array}
$$

where prime denotes differentiation with respect to $\eta$, and $a$ is a positive constant. Also we denote $u_{r}(x)=a x$ as a reference velocity for this problem and $u=u_{r}(x) f^{\prime}(\eta)$. Using (3) and the boundary conditions (6), we obtain the following expression for the pressure $P$ :

$$
P=P_{0}-\rho \frac{v^{2}}{2}+\rho v \frac{d v}{d y}-\frac{\rho v}{k^{\prime}} \int v d y,
$$


where $P_{0}$ is the stagnation pressure. Substituting (7) and (8) into (2), (4), and (5), we obtain the following nonlinear ordinary differential equations:

$$
\begin{aligned}
& f^{\prime \prime \prime}+f f^{\prime \prime}-f^{\prime 2}-K f^{\prime}=0, \\
& \theta^{\prime \prime}+\operatorname{Pr} f \theta^{\prime}+\operatorname{Pr} D_{f} \phi^{\prime \prime}=0, \\
& \phi^{\prime \prime}+\operatorname{Sc} f \phi^{\prime}+\operatorname{ScSr} \theta^{\prime \prime}=0,
\end{aligned}
$$

subject to the boundary conditions

$$
\begin{gathered}
f(0)=s, \quad f^{\prime}(0)=\frac{b}{a}=\alpha, \\
\theta(0)=1, \quad \phi(0)=1, \\
f^{\prime}(\infty)=0, \quad \theta(\infty)=0, \quad \phi(\infty)=0,
\end{gathered}
$$

where $K=\left(v / k^{\prime} a\right)$ is the permeability parameter, $\operatorname{Pr}$ is the Prandtl number of the fluid with $\operatorname{Pr}=v / \sigma, D_{f}=\left(D_{m} k_{T}\left(C_{w}-\right.\right.$ $\left.\left.C_{\infty}\right) / c_{s} c_{p} v\left(T_{w}-T_{\infty}\right)\right)$ is the Dufour number, Sc $=\left(v / D_{m}\right)$ is the Schmidt number, $\mathrm{Sr}=\left(D_{m} k_{T}\left(T_{w}-T_{\infty}\right) / v T_{m}\left(C_{w}-C_{\infty}\right)\right)$ is the Soret number, and $s$ is the mass transfer parameter showing the strength of the mass transfer at the sheet. Also $\alpha>0$ is for wall stretching and $\alpha<0$ is for shrinking, respectively.

A physical quantity of interest is the skin friction coefficient $C_{f}$, which is defined as

$$
C_{f}=\frac{\tau_{w}}{\rho u_{r}^{2}}
$$

where $\tau_{w}$ is the shear stress and is given by

$$
\tau_{w}=\mu\left(\frac{\partial u}{\partial y}\right)_{y=0}
$$

Using the similarity variables (7), we obtain

$$
\operatorname{Re}_{x}^{1 / 2} C_{f}=f^{\prime \prime}(0),
$$

where $\operatorname{Re}_{x}=u_{r} x / v$ is the local Reynolds number.

The momentum equation (9) together with the boundary conditions (12) yields an algebraically decaying solution as follows:

$$
\begin{gathered}
f(\eta)=\frac{-6 \alpha}{\sqrt{6(K-\alpha)}+(K-\alpha) \eta}, \\
f^{\prime}(\eta)=\frac{6 \alpha(K-\alpha)}{(\sqrt{6(K-\alpha)}+(K-\alpha) \eta)^{2}} .
\end{gathered}
$$

The velocity fields become

$$
\begin{gathered}
u=\frac{6 b x(K-\alpha)}{(\sqrt{6(K-\alpha)}+(K-\alpha) \eta)^{2}}, \\
v=\frac{6 \alpha \sqrt{a v}}{\sqrt{6(K-\alpha)}+(K-\alpha) \eta} .
\end{gathered}
$$

It is seen that the algebraically decaying function exists only for a shrinking sheet with $\alpha<0$. The mass suction at the sheet is

$$
f(0)=s=\frac{-6 \alpha}{\sqrt{6(K-\alpha)}} .
$$

There exists another exact solution for (9) with the boundary conditions (12) as

$$
f(\eta)=s+\frac{\alpha}{\delta}\left(1-e^{-\delta \eta}\right)
$$

with

$$
\delta=\frac{s \pm \sqrt{s^{2}+4(K+\alpha)}}{2}
$$

Eliminating $\phi^{\prime \prime}$ from (10) and (11), we obtain

$$
\left(1-\operatorname{Pr} D_{f} \operatorname{Sc} \operatorname{Sr}\right) \frac{1}{f} \theta^{\prime \prime}+\operatorname{Pr} \theta^{\prime}-\operatorname{Pr} D_{f} S c \phi^{\prime}=0 .
$$

Differentiating (20) with respect to $\eta$ :

$$
\begin{aligned}
& \left(1-\operatorname{Pr} D_{f} \operatorname{ScSr}\right) \frac{1}{f} \theta^{\prime \prime \prime}+\left[\frac{\left(1-\operatorname{Pr} D_{f} \mathrm{ScSr}\right)(K-\alpha)}{-6 \alpha}+\operatorname{Pr}\right] \theta^{\prime \prime} \\
& -\operatorname{Pr} D_{f} \operatorname{Sc} \phi^{\prime \prime}=0 .
\end{aligned}
$$

Eliminating $\phi^{\prime \prime}$ between (10) and (21), we obtain the EulerCauchy equation:

$$
\begin{aligned}
\frac{1}{f^{3}} \theta^{\prime \prime \prime} & +\left[\frac{K-\alpha}{-6 \alpha}+\frac{\operatorname{Pr}+\mathrm{Sc}}{1-\operatorname{Pr} D_{f} \mathrm{ScSr}}\right] \frac{1}{f^{2}} \theta^{\prime \prime} \\
& +\left(\frac{\operatorname{Pr} \mathrm{Sc}}{1-\operatorname{Pr} D_{f} \mathrm{ScSr}}\right) \frac{1}{f} \theta^{\prime}=0 .
\end{aligned}
$$

Or,

$$
\begin{aligned}
& \left(\frac{\sqrt{6(K-\alpha)}+(K-\alpha) \eta}{-6 \alpha}\right)^{3} \theta^{\prime \prime \prime}+\left[\frac{K-\alpha}{-6 \alpha}+\frac{\operatorname{Pr}+\mathrm{Sc}}{1-\operatorname{Pr} D_{f} \mathrm{Sc} \mathrm{Sr}}\right] \\
& \quad \times\left(\frac{\sqrt{6(K-\alpha)}+(K-\alpha) \eta}{-6 \alpha}\right)^{2} \theta^{\prime \prime}+\left(\frac{\operatorname{PrSc}}{1-\operatorname{Pr} D_{f} \mathrm{Sc} \mathrm{Sr}}\right) \\
& \quad \times\left(\frac{\sqrt{6(K-\alpha)}+(K-\alpha) \eta}{-6 \alpha}\right) \theta^{\prime}=0 .
\end{aligned}
$$

The solution can be found as follows:

$$
\begin{aligned}
\theta(\eta)= & c_{1}\left[\frac{-6 \alpha}{\sqrt{6(K-\alpha)}+(K-\alpha) \eta}\right]^{A_{1}} \\
& +c_{2}\left[\frac{-6 \alpha}{\sqrt{6(K-\alpha)}+(K-\alpha) \eta}\right]^{A_{2}}+c_{3},
\end{aligned}
$$




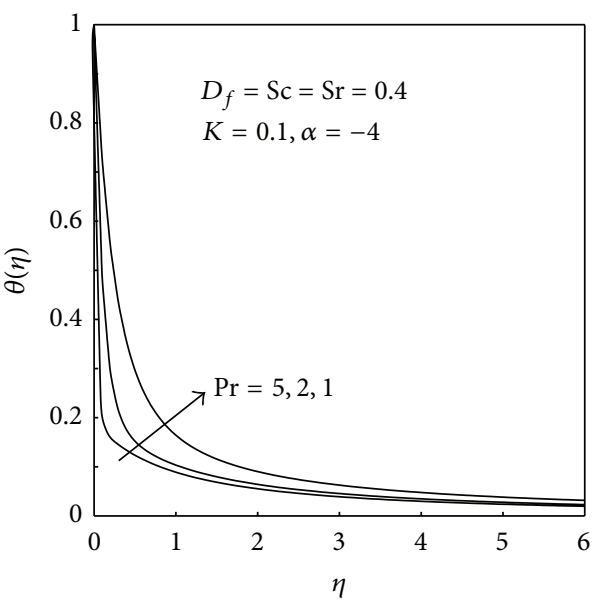

(a)

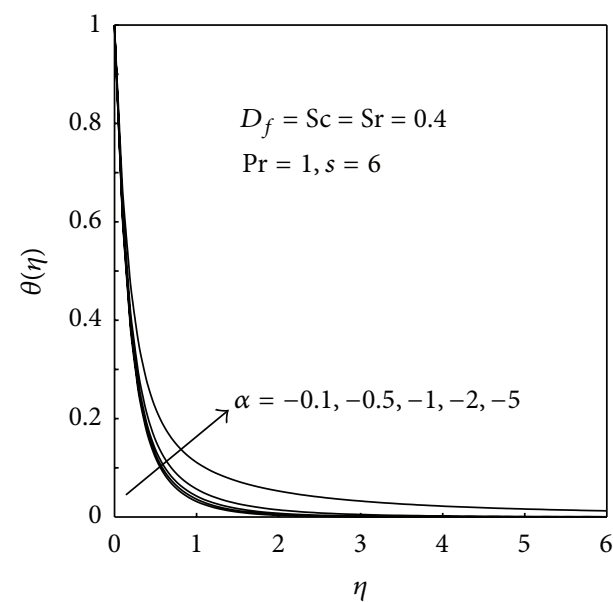

(c)

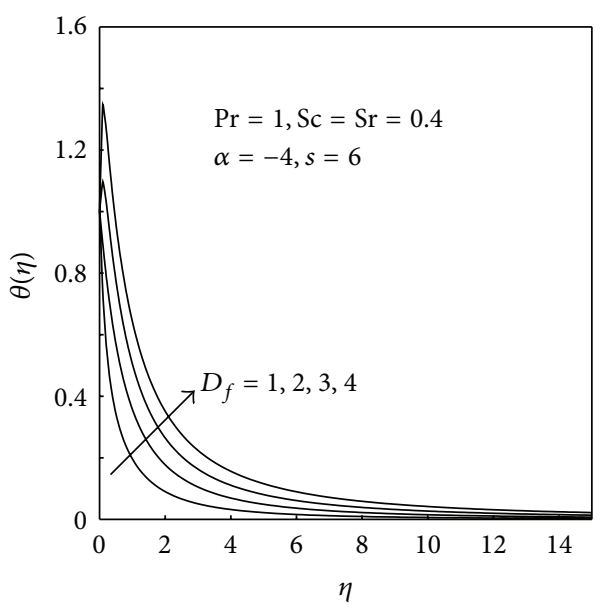

(e)

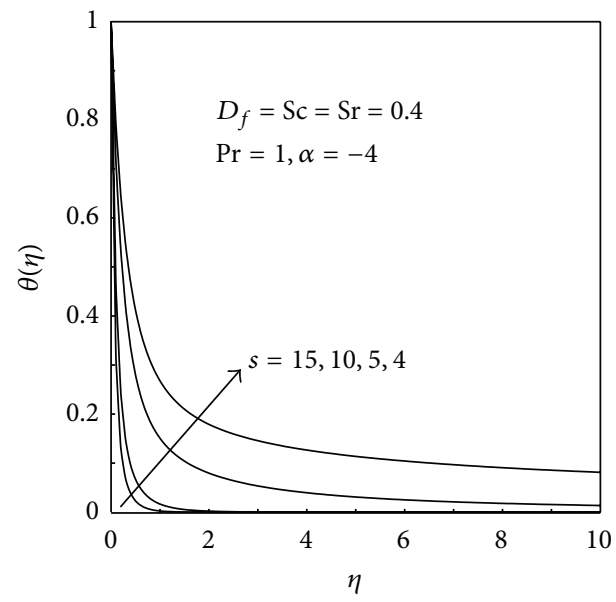

(b)

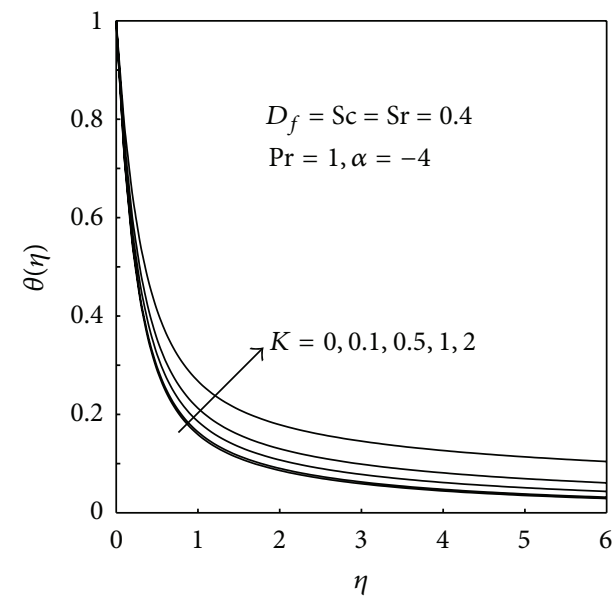

(d)

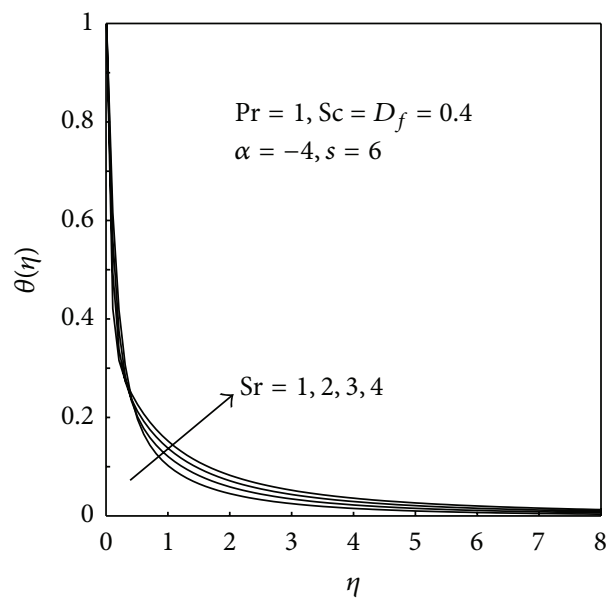

(f)

Figure 2: (a) Temperature profiles for a shrinking sheet problem under different values of Prandtl number. (b) Effects of the mass transfer parameter (mass suction) on the temperature profiles of a shrinking sheet. (c) Temperature profiles for different values of shrinking sheet strength with mass suction. (d) Effects of the permeability parameter on the temperature profiles of a shrinking sheet with mass suction. (e) Effects of Dufour number $D_{f}$ on the temperature profiles of a shrinking sheet with mass suction. (f) Effects of Soret number Sr on the temperature profiles of a shrinking sheet with mass suction. 


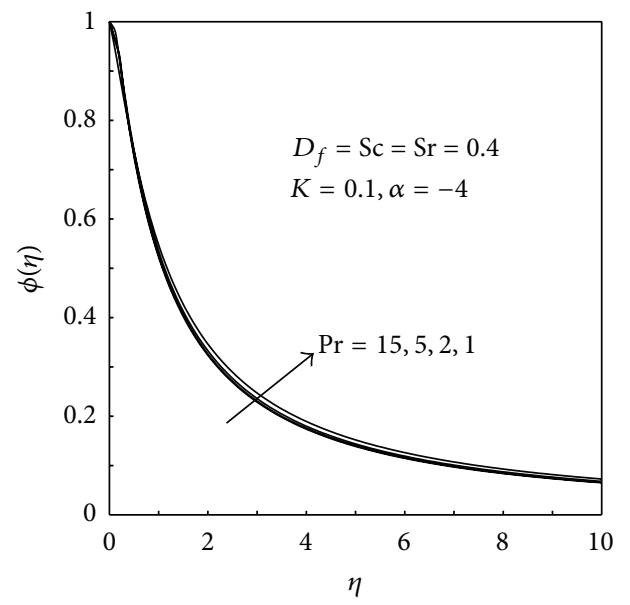

(a)

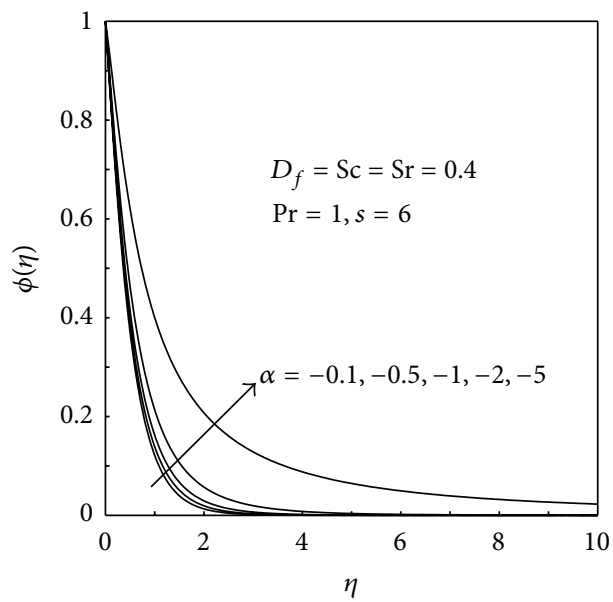

(c)

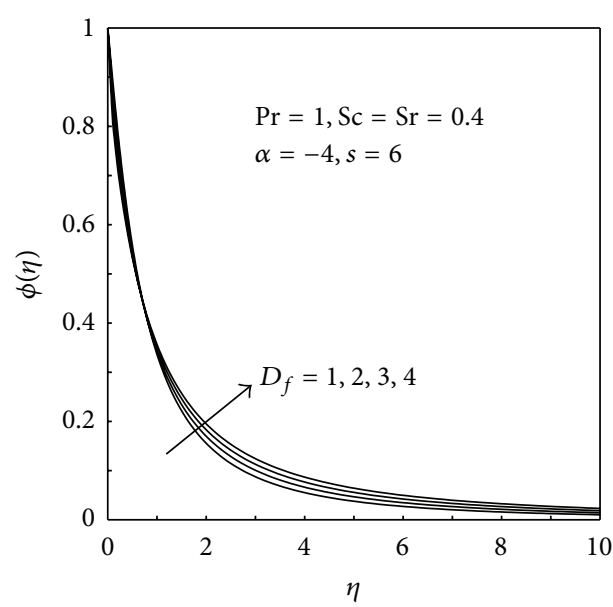

(e)

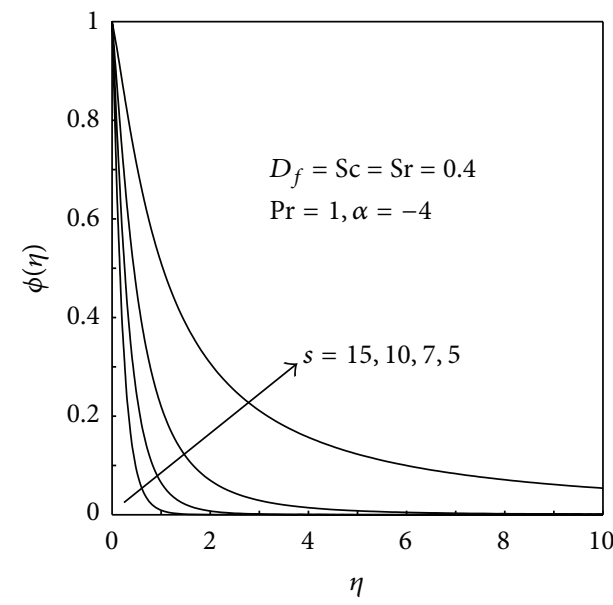

(b)

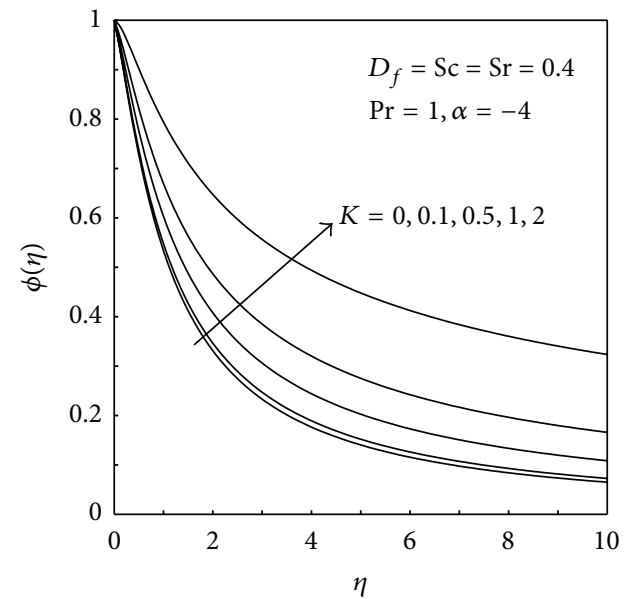

(d)

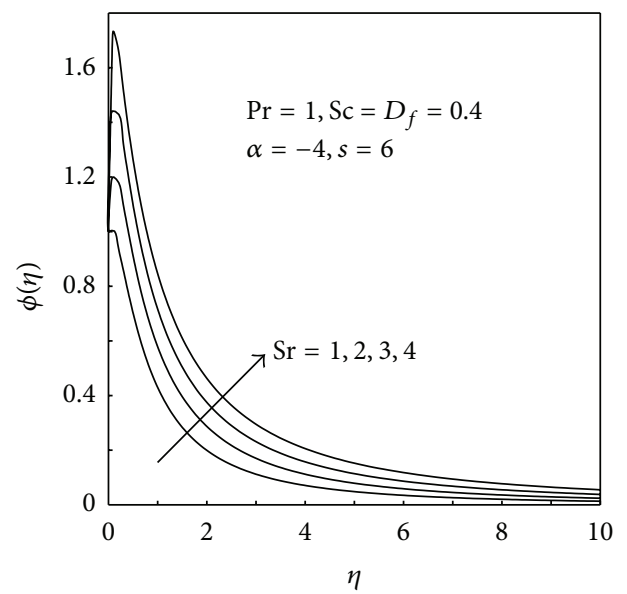

(f)

FIGURE 3: (a) Concentration profiles for a shrinking sheet under different values of Prandtl number. (b) Effects of the mass transfer parameter (mass suction) on the concentration profiles of a shrinking sheet. (c) Concentration profiles for different values of shrinking sheet strength with mass suction. (d) Effects of the permeability parameter on the concentration profiles of a shrinking sheet with mass suction. (e) Effects of Dufour number $D_{f}$ on the concentration profiles of a shrinking sheet with mass suction. (f) Effects of Soret number Sr on the concentration profiles of a shrinking sheet with mass suction. 


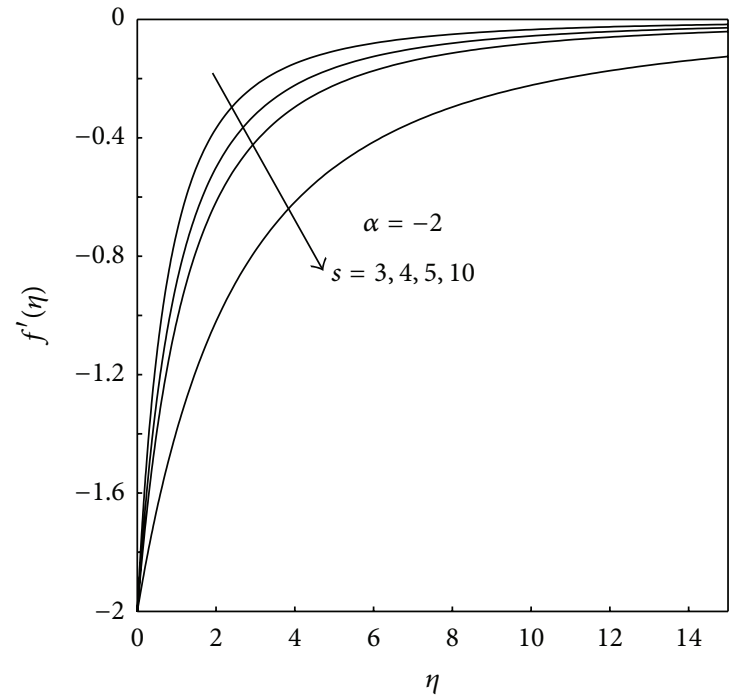

(a)

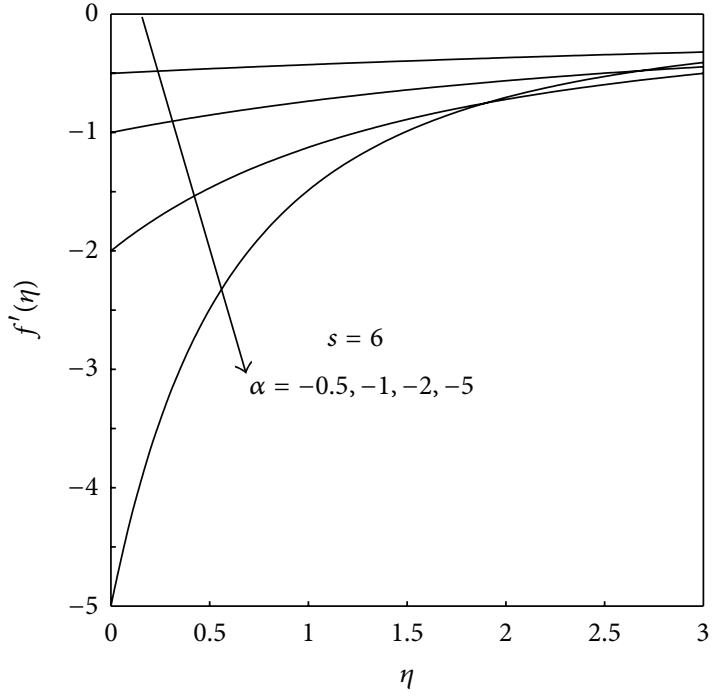

(b)

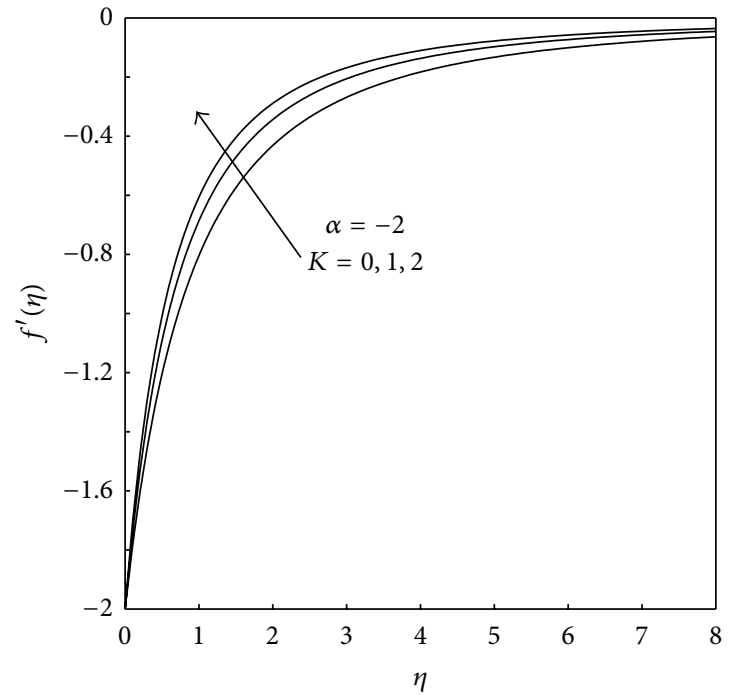

(c)

Figure 4: (a) Velocity profiles for a shrinking sheet under different values of mass suction parameter. (b) Velocity profiles under different values of shrinking sheet strength with mass suction. (c) Velocity profiles for a shrinking sheet under different values of permeability parameter.

where

$$
\begin{aligned}
A_{1}= & \left(\frac{-3 \alpha}{K-\alpha}\right)\left(\frac{\mathrm{Pr}+\mathrm{Sc}}{1-\mathrm{Pr} D_{f} \mathrm{ScSr}}\right) \\
& \times\left(1-\sqrt{\frac{(\mathrm{Pr}-\mathrm{Sc})^{2}+4 \mathrm{Pr}^{2} \mathrm{Sc}^{2} D_{f} \mathrm{Sr}}{(\mathrm{Pr}+\mathrm{Sc})^{2}}}\right)-1 \\
A_{2}= & \left(\frac{-3 \alpha}{K-\alpha}\right)\left(\frac{\mathrm{Pr}+\mathrm{Sc}}{1-\mathrm{Pr} D_{f} \mathrm{ScSr}}\right) \\
& \times\left(1+\sqrt{\frac{(\mathrm{Pr}-\mathrm{Sc})^{2}+4 \mathrm{Pr}^{2} \mathrm{Sc}^{2} D_{f} \mathrm{Sr}}{\left(\mathrm{Pr}+\mathrm{Sc}^{2}\right.}}\right)-1 .
\end{aligned}
$$

In order to match the $\mathrm{BC}$ at $\eta \rightarrow \infty$, it is required that $A_{1}>0$ and $A_{2}>0$, and $c_{3}=0$

$$
\begin{aligned}
\theta(\eta)= & c_{1}\left[\frac{-6 \alpha}{\sqrt{6(K-\alpha)}+(K-\alpha) \eta}\right]^{A_{1}} \\
& +c_{2}\left[\frac{-6 \alpha}{\sqrt{6(K-\alpha)}+(K-\alpha) \eta}\right]^{A_{2}}, \\
\theta^{\prime}(\eta)= & c_{1} A_{1}\left(\frac{K-\alpha}{6 \alpha}\right)\left[\frac{-6 \alpha}{\sqrt{6(K-\alpha)}+(K-\alpha) \eta}\right]^{A_{1}+1} \\
& +c_{2} A_{2}\left(\frac{K-\alpha}{6 \alpha}\right)\left[\frac{-6 \alpha}{\sqrt{6(K-\alpha)}+(K-\alpha) \eta}\right]^{A_{2}+1}
\end{aligned}
$$




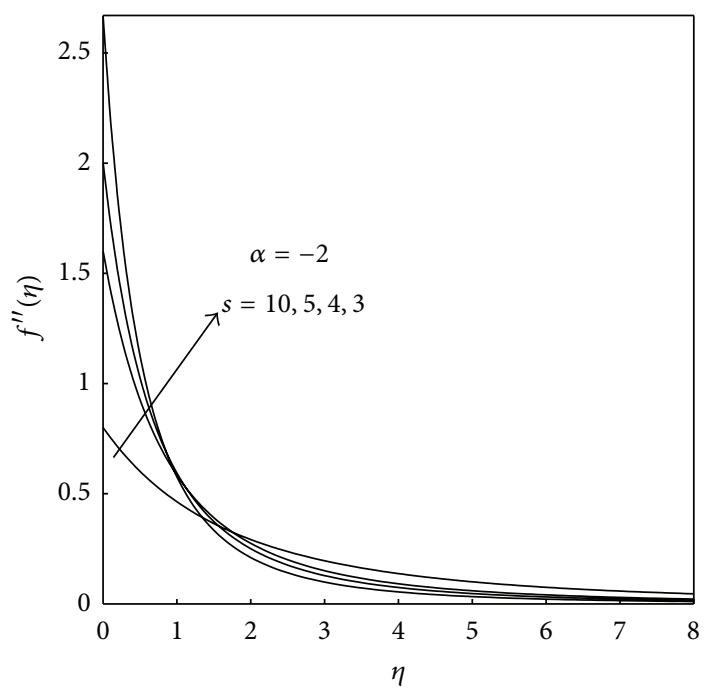

(a)

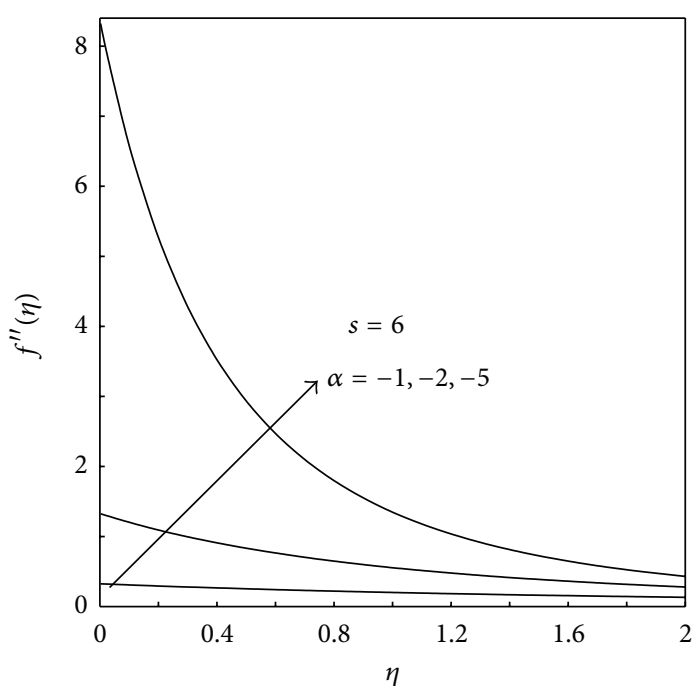

(b)

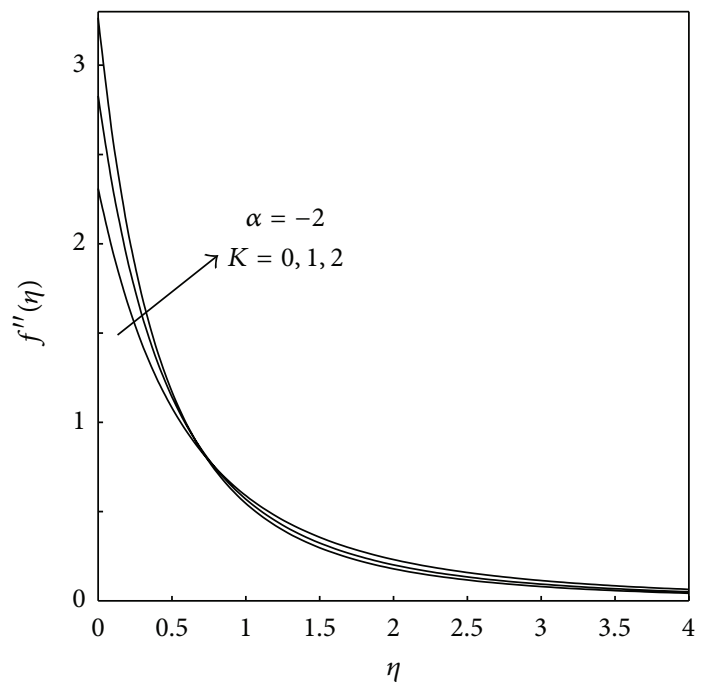

(c)

FIGURE 5: (a) Shear stress profiles for a shrinking sheet under different values of mass suction parameter. (b) Shear stress profiles under different values of shrinking sheet strength with mass suction. (c) Shear stress profiles for a shrinking sheet under different values of permeability parameter.

Integrating (20) with respect to $\eta$ :

$$
\begin{aligned}
\phi(\eta)= & \left(\frac{1-\operatorname{Pr} D_{f} \mathrm{ScS} S}{\operatorname{Pr} D_{f} \mathrm{Sc}}\right) \frac{1}{f} \theta^{\prime} \\
& +\left(\frac{\left(1-\operatorname{Pr} D_{f} \mathrm{ScS}\right)((K-\alpha) / 6 \alpha)+\operatorname{Pr}}{\operatorname{Pr} D_{f} \mathrm{Sc}}\right) \theta-\frac{c_{0}}{\operatorname{Pr} D_{f} \mathrm{Sc}},
\end{aligned}
$$

where $c_{0}$ is a constant.
Substituting from (26) into (27), we obtain

$$
\begin{aligned}
\phi(\eta)= & c_{1} A_{3}\left[\frac{-6 \alpha}{\sqrt{6(K-\alpha)}+(K-\alpha) \eta}\right]^{A_{1}} \\
& +c_{2} A_{4}\left[\frac{-6 \alpha}{\sqrt{6(K-\alpha)}+(K-\alpha) \eta}\right]^{A_{2}}-\frac{c_{0}}{\operatorname{Pr} D_{f} S c}
\end{aligned}
$$

where

$$
\begin{aligned}
& A_{3}=\left(\frac{1-\operatorname{Pr} D_{f} \mathrm{ScSr}}{\operatorname{Pr} D_{f} \mathrm{Sc}}\right)\left(\frac{K-\alpha}{6 \alpha}\right)\left(A_{1}+1\right)+\frac{1}{D_{f} \mathrm{Sc}}, \\
& A_{4}=\left(\frac{1-\operatorname{Pr} D_{f} \mathrm{ScSr}}{\operatorname{Pr} D_{f} \mathrm{Sc}}\right)\left(\frac{K-\alpha}{6 \alpha}\right)\left(A_{2}+1\right)+\frac{1}{D_{f} \mathrm{Sc}} .
\end{aligned}
$$




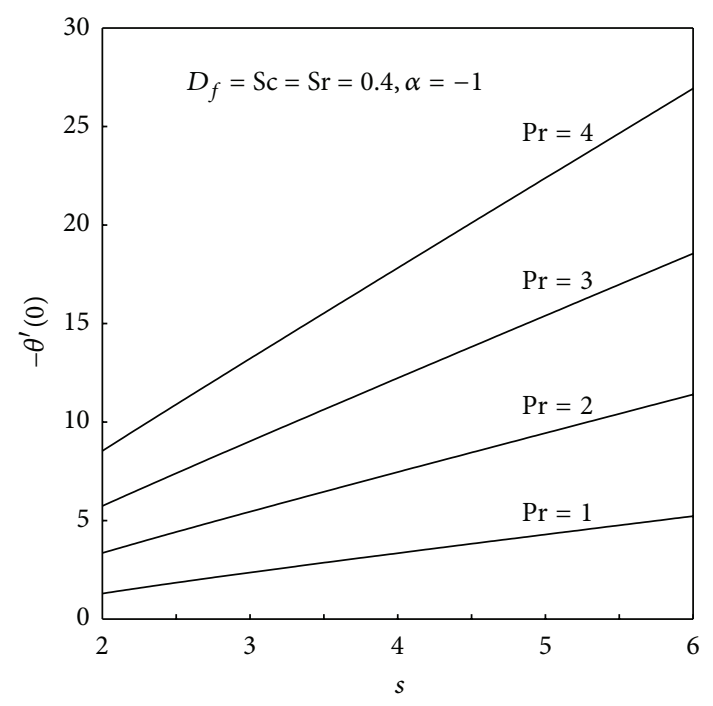

(a)

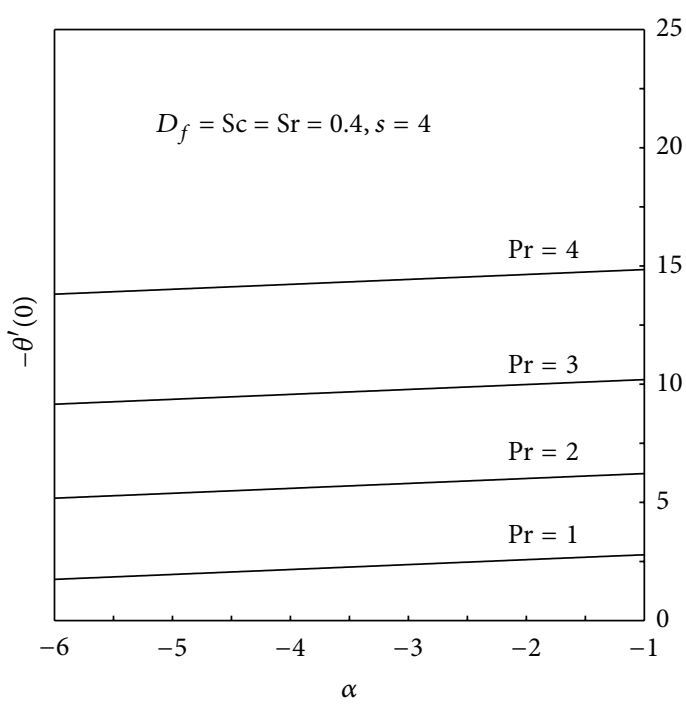

(b)

Figure 6: (a) Wall heat flux, $-\theta^{\prime}(0)$ with $s$ for various values of Pr. (b) Wall heat flux, $-\theta^{\prime}(0)$ with $\alpha$ for various values of Pr.

In order to match the boundary conditions at $\eta \rightarrow \infty$, it is required that $A_{1}>0$ and $A_{2}>0$, and $c_{0}=0$, so (28) becomes

$$
\begin{aligned}
\phi(\eta)= & c_{1} A_{3}\left[\frac{-6 \alpha}{\sqrt{6(K-\alpha)}+(K-\alpha) \eta}\right]^{A_{1}} \\
& +c_{2} A_{4}\left[\frac{-6 \alpha}{\sqrt{6(K-\alpha)}+(K-\alpha) \eta}\right]^{A_{2}} .
\end{aligned}
$$

Applying the boundary conditions at the wall yields

$$
\begin{gathered}
c_{1}\left(\frac{-6 \alpha}{\sqrt{6(K-\alpha)}}\right)^{A_{1}}+c_{2}\left(\frac{-6 \alpha}{\sqrt{6(K-\alpha)}}\right)^{A_{2}}=1, \\
c_{1} A_{3}\left(\frac{-6 \alpha}{\sqrt{6(K-\alpha)}}\right)^{A_{1}}+c_{2} A_{4}\left(\frac{-6 \alpha}{\sqrt{6(K-\alpha)}}\right)^{A_{2}}=1 .
\end{gathered}
$$

Solving (31), we obtain

$$
\begin{aligned}
& c_{1}=\frac{1-A_{4}}{A_{3}-A_{4}}\left(\frac{\sqrt{6(K-\alpha)}}{-6 \alpha}\right)^{A_{1}}, \\
& c_{2}=\frac{1-A_{3}}{A_{4}-A_{3}}\left(\frac{\sqrt{6(K-\alpha)}}{-6 \alpha}\right)^{A_{2}} .
\end{aligned}
$$

Then the temperature and concentration solutions become

$$
\begin{aligned}
\theta(\eta)= & \frac{1-A_{4}}{A_{3}-A_{4}} \frac{1}{(\sqrt{((K-\alpha) / 6)} \eta+1)^{A_{1}}} \\
& +\frac{1-A_{3}}{A_{4}-A_{3}} \frac{1}{(\sqrt{((K-\alpha) / 6)} \eta+1)^{A_{2}}}, \\
\phi(\eta)= & \frac{A_{3}\left(1-A_{4}\right)}{A_{3}-A_{4}} \frac{1}{(\sqrt{((K-\alpha) / 6)} \eta+1)^{A_{1}}} \\
& +\frac{A_{4}\left(1-A_{3}\right)}{A_{4}-A_{3}} \frac{1}{(\sqrt{((K-\alpha) / 6)} \eta+1)^{A_{2}}} .
\end{aligned}
$$

The heat and mass transfer rates at the wall are related to the temperature and concentration gradients at the wall are, respectively,

$$
\begin{gathered}
q_{w}=-k\left(\frac{\partial T}{\partial y}\right)_{y=0}=-k\left(T_{w}-T_{\infty}\right) \sqrt{\frac{a}{v}} \theta^{\prime}(0), \\
m_{w}=-D_{m}\left(\frac{\partial C}{\partial y}\right)_{y=0}=-D_{m}\left(C_{w}-C_{\infty}\right) \sqrt{\frac{a}{v}} \phi^{\prime}(0),
\end{gathered}
$$

where $k$ is the thermal conductivity of the fluid.

The heat and mass fluxes at the wall read

$$
\begin{aligned}
& \left.-\theta^{\prime}(0)=\sqrt{\frac{K-\alpha}{6}}\left[\frac{A_{1}\left(1-A_{4}\right)-A_{2}\left(1-A_{3}\right)}{A_{3}-A_{4}}\right], \quad \text { (35a }\right) \\
& -\phi^{\prime}(0)=\sqrt{\frac{K-\alpha}{6}}\left[\frac{A_{1} A_{3}\left(1-A_{4}\right)-A_{2} A_{4}\left(1-A_{3}\right)}{A_{3}-A_{4}}\right] .
\end{aligned}
$$




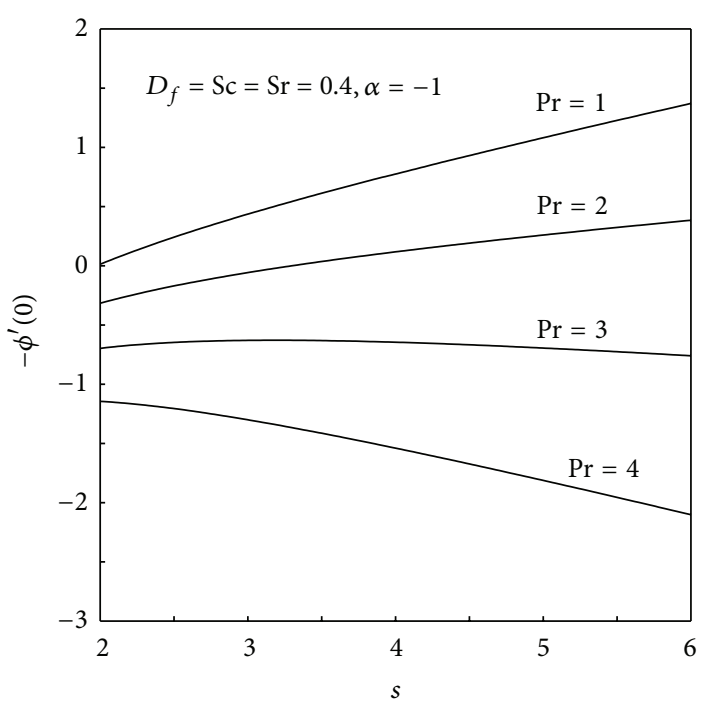

(a)

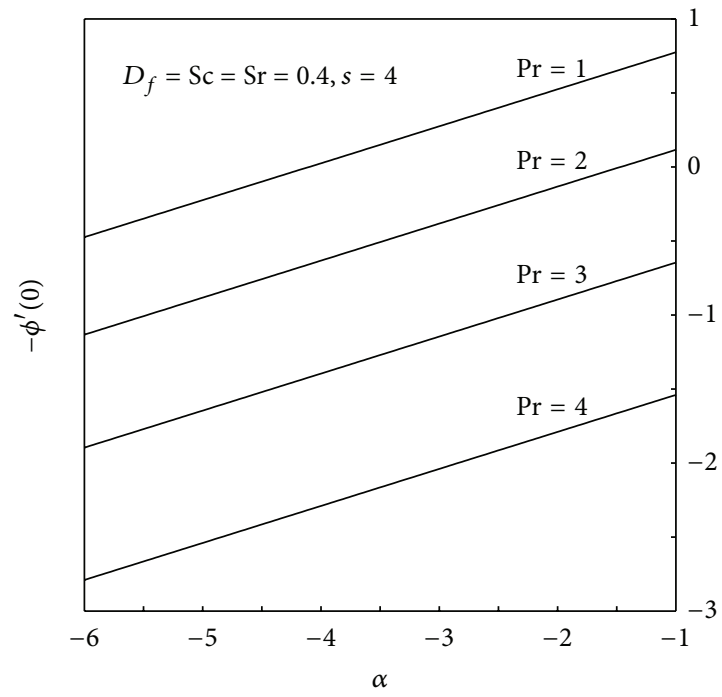

(b)

Figure 7: (a) Wall mass flux, $-\phi^{\prime}(0)$ with $s$ for various values of Pr. (b) Wall mass flux, $-\phi^{\prime}(0)$ with $\alpha$ for various values of Pr.

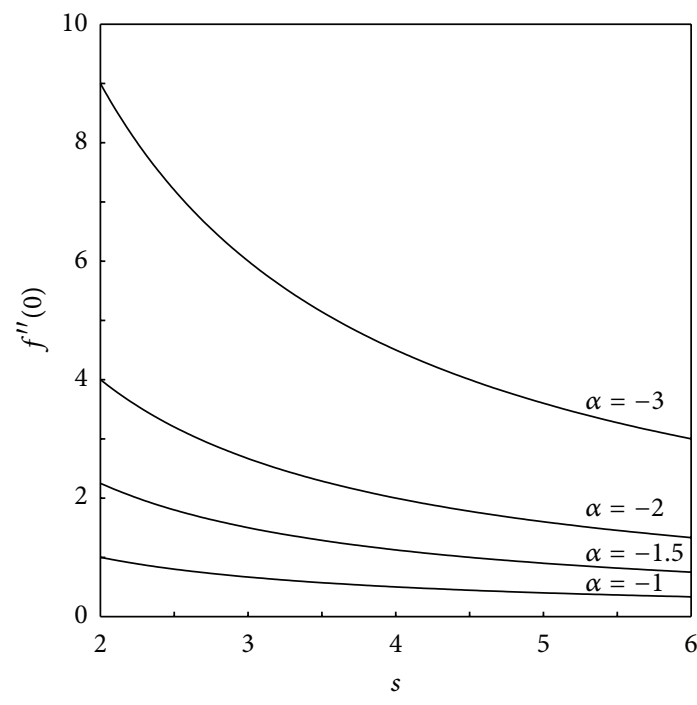

(a)

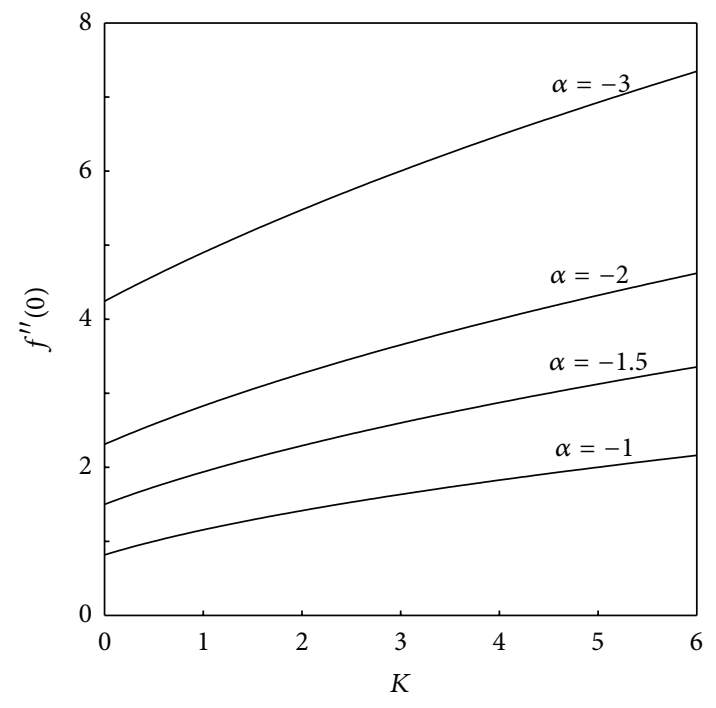

(b)

FIGURE 8: (a) Wall shear stress, $f^{\prime \prime}(0)$ with $s$ for various values of $\alpha$. (b) Wall shear stress, $f^{\prime \prime}(0)$ with $K$ for various values of $\alpha$.

\section{Results and Discussion}

The nondimensional temperature profiles for a shrinking sheet are shown in Figures 2(a)-2(f). The effect of the Prandtl number on the temperature profile is shown in Figure 2(a). It is observed that the temperature increases with decreasing values of the Prandtl number. With the increase of Prandtl number, the temperature drops faster and the boundary layer becomes thinner. Figure 2(b) illustrates the influence of the mass transfer parameter (mass suction) for a shrinking sheet problem on the temperature profile. It is found that the temperature increases with the decrease of suction parameter. With the increase in mass suction, the temperature drops faster and the boundary layer becomes thinner. Figure 2(c) represents the temperature profile for different values of shrinking sheet strength. It is noticed from the figure that the temperature decreases with decreasing the magnitude of shrinking sheet strength. The boundary layer becomes thicker for a high magnitude of shrinking strength. Figure 2(d) represents the temperature profile for different values of Permeability parameter $K$. From the graph we observe that the increase of Permeability parameter leads to the increase of the temperature profile. The temperature distribution for different values of $D_{f}$ is plotted in Figure 2(e). 
It is observed from this figure that the temperature distribution is more for higher values of $D_{f}$ which increases with an increase in the value of $D_{f}$. Higher values of $D_{f}$ gives rise to the formation of peak near the sheet. Figure 2(f) shows the variation of concentration profiles for various values of Soret number Sr. We notice that for $\eta \geq 0.5$, the temperature distribution increases with increasing $\mathrm{Sr}$.

Figure 3(a) displays the effect of Prandtl number on the concentration profile under certain shrinking strength, and Permeability parameter. It can be seen that for $\eta \geq 1.3$, the concentration increases when the Prandtl number decreases. The concentration boundary layer thickness reduces with the increase of the Prandtl number. The effect of the mass transfer parameter (mass suction) on the dimensionless concentration profile for sheet shrinking problem is shown in Figure 3(b). We observe that the concentration increases with the decrease of mass suction. The concentration boundary layer thickness decreases with the increase of mass transfer parameter (mass suction). Figure 3(c) represents the concentration profile for different values of shrinking sheet strength. From the graph we observe that the concentration decreases with decreasing the magnitude of shrinking sheet strength and the concentration boundary layer thickness becomes thinner. Figure 3(d) displays the concentration profile for different values of Permeability parameter. We notice from the figure that the effect of increasing values of Permeability parameter is to increase the concentration profile. Figure 3(e) shows the concentration profiles for different values of Dufour number $D_{f}$. It is observed that for $\eta \geq$ 0.8 , the concentration distribution increases with increasing the Dufour number $D_{f}$. The concentration distribution for different values of Soret number Sr is plotted in Figure 3(f). It is noticed from this figure that the temperature distribution is more for higher values of Sr which increases with an increase in the value of $\mathrm{Sr}$ and peaks appear near the sheet for higher values of $\mathrm{Sr}$.

The effect of mass suction parameter on the velocity profile for shrinking sheet is displayed in Figure 4(a) which shows that the velocity decreases with the increase of the mass suction parameter. The boundary layer is sucked closer to the wall for smaller mass suction parameters. Figure 4(b) displays the velocity profile for various values of shrinking sheet parameter. The velocity decreases and drops faster with high magnitude of sheet shrinking parameter $\alpha$. The velocity at the sheet decreases with increasing the magnitude of shrinking sheet parameter $\alpha$. There exists cross-over points among the velocity profiles for different values of $\alpha$. Figure $4(\mathrm{c})$ shows velocity profile for different values of the permeability parameter for shrinking sheet. We remark that as permeability parameter increases the velocity decreases.

Shear stress results for shrinking sheet are seen in Figure 5(a) under the effect of mass suction. The shear stress drops faster as the mass suction becomes smaller. The increasing effect of shear stress is found in the interval $0 \leq \eta \leq 1$ with decreasing $s$. The wall shear stress decreases with increasing mass suction. There exists cross-over points among the shear profiles for different values of $s$. In Figure 5(b) the shear stress increases with increasing magnitude of shrinking sheet parameter. The shear drops faster when magnitude of shrinking sheet parameter increases and the wall shear stress decreases with decreasing magnitude of shrinking parameter. Figure 5(c) illustrates the shear stress profile for a shrinking sheet with mass suction. The wall shear stress increases permeability parameter. The increasing effect of shear stress is found in the interval $0 \leq \eta \leq 0.8$ with increasing $K$.

Figures 6(a) and 6(b) and Figures 7(a) and 7(b) show the rate of heat and mass transfer at the sheet surface versus mass transfer parameter $s$ and sheet shrinking parameter $\alpha$, respectively, with different values of Prandtl number Pr. We observe that $-\theta^{\prime}(0)$ increase with increasing the values of $\mathrm{Pr}$ whereas reverse effect is observed on $-\phi^{\prime}(0)$.

Figures 8(a) and 8(b) depict the effect of sheet shrinking parameter $\alpha$ on wall shear stress $f^{\prime \prime}(0)$ versus mass suction $s$ and permeability parameter $K$, respectively. We notice that $f^{\prime \prime}(0)$ increase with increasing the magnitude of shrinking sheet parameter $\alpha$.

\section{Conclusion}

In this work, the solutions for the momentum, heat, and mass transfer equations are obtained in a closed analytical form and the solutions presented in terms of algebraically decaying function. The effects of the Prandtl number, the mass transfer parameter, the wall shrinking parameter, and the Permeability parameter on the temperature and concentration profiles were analyzed. Also, the effects of mass suction, Permeability of the porous medium, and shrinking strength on the velocity and shear stress are studied. We found some of the important results from the graphical representation which are listed below.

(1) The effect of Prandtl number Pr is to reduce the thickness of both the temperature and the concentration boundary layer.

(2) The effect of mass transfer parameter $s$ (mass suction) is seen to decrease both of the temperature and the concentration distributions in the flow region.

(3) Increasing magnitude of shrinking sheet strength is to increase both of the temperature and the concentration distributions in the flow region

(4) The effect of Permeability parameter $K$ is seen to increase both of the temperature and the concentration distributions in the flow region.

(5) Dufour effect is to increase both temperature and concentration distributions with formation of peak for higher values of Dufour parameter in the thermal boundary layer.

(6) Soret effect is to increase both temperature and concentration distributions with formation of peak for higher values of Soret parameter in the concentration boundary layer.

(7) The velocity decreases with the increase of the mass suction parameter $s$, the magnitude of shrinking sheet strength $\alpha$, and the Permeability parameter $K$.

(8) The shear stress increases with the increase of the mass suction parameter $s$, magnitude of shrinking 
sheet strength parameter $\alpha$, and Permeability parameter $K$.

(9) $-\theta^{\prime}(0)$ versus $s$, increases with increasing the values of Pr whereas reverse effect is observed on $-\phi^{\prime}(0)$. Also, $-\theta^{\prime}(0)$ versus $\alpha$ increases with increasing the values of Pr whereas reverse effect is observed on $-\phi^{\prime}(0)$.

(10) $f^{\prime \prime}(0)$ increases with increasing the magnitude of shrinking sheet parameter $\alpha$.

\section{References}

[1] B. C. Sakiadis, "Boundary-layer behavior on continuous solid surface: I. Boundary-layer equations for two-dimensional and axisymmetric flow," AIChE Journal, vol. 7, pp. 26-28, 1961.

[2] B. C. Sakiadis, "Boundary-layer behavior on continuous solid surface: II. Boundary-layer equations for two-dimensional and axisymmetric flow," AIChE Journal, vol. 7, pp. 221-225, 1961.

[3] L. J. Crane, "Flow past a stretching plate," Journal of Applied Mathematics and Physics, vol. 21, no. 4, pp. 645-647, 1970.

[4] P. S. Gupta and A. S. Gupta, "Heat and mass transfer on a stretching sheet with suction or blowing," The Canadian Journal of Chemical Engineering, vol. 55, pp. 744-746, 1977.

[5] C. K. Chen and M. I. Char, "Heat transfer of a continuous, stretching surface with suction or blowing," Journal of Mathematical Analysis and Applications, vol. 135, no. 2, pp. 568-580, 1988.

[6] M. Ferdows, M. Ota, A. Sattar, and M. Alam, "Similarity solutions for MHD through vertical porous plate with suction," Journal of Computational and Applied Mathematics, vol. 6, pp. 15-25, 2005.

[7] J. R. Lin, L. J. Liang, and R. D. Chien, "Magneto-hydrodynamic flow of a second order fluid over a stretching sheet with suction," Journal of the Chinese Institute of Engineers, vol. 30, no. 1, pp. 183-188, 2007.

[8] P. S. Gupta and A. S. Gupta, "Heat and mass transfer on a stretching sheet with suction or blowing," The Canadian Journal of Chemical Engineering, vol. 55, pp. 744-746, 1977.

[9] C. K. Chen and M. I. Char, "Heat transfer of a continuous, stretching surface with suction or blowing," Journal of Mathematical Analysis and Applications, vol. 135, no. 2, pp. 568-580, 1988.

[10] R. S. R. Gorla, D. E. Abboud, and A. Sarmah, "Magnetohydrodynamic flow over a vertical stretching surface with suction and blowing," Heat and Mass Transfer, vol. 34, no. 2-3, pp. 121-125, 1998.

[11] S. Yao, T. Fang, and Y. Zhong, "Heat transfer of a generalized stretching/shrinking wall problem with convective boundary conditions," Communications in Nonlinear Science and Numerical Simulation, vol. 16, no. 2, pp. 752-760, 2011.

[12] T. Fang, S. Yao, and I. Pop, "Flow and heat transfer over a generalized stretching/shrinking wall problem-Exact solutions of the Navier-Stokes equations," International Journal of NonLinear Mechanics, vol. 46, pp. 1116-1127, 2011.

[13] T. Fang, "Boundary layer flow over a shrinking sheet with power-law velocity," International Journal of Heat and Mass Transfer, vol. 51, no. 25-26, pp. 5838-5843, 2008.

[14] T. Fang, W. Liang, and C. F. Lee, "A new solution branch for the Blasius equation - shrinking sheet problem," Computers \& Mathematics with Applications, vol. 56, no. 12, pp. 3088-3095, 2008.
[15] T. Fang and J. Zhang, "Closed-form exact solutions of MHD viscous flow over a shrinking sheet," Communications in Nonlinear Science and Numerical Simulation, vol. 14, no. 7, pp. 2853-2857, 2009.

[16] T. Fang, J. Zhang, and S. Yao, "Slip MHD viscous flow over a stretching sheet-an exact solution," Communications in Nonlinear Science and Numerical Simulation, vol. 14, no. 11, pp. 3731-3737, 2009.

[17] M. Miklavčič and C. Y. Wang, "Viscous flow due to a shrinking sheet," Quarterly of Applied Mathematics, vol. 64, no. 2, pp. 283290, 2006.

[18] C. Y. Wang, "Stagnation flow towards a shrinking sheet," International Journal of Non-Linear Mechanics, vol. 43, no. 5, pp. 377-382, 2008.

[19] M. S. Alam and M. M. Rahman, "Dufour and Soret effects on mixed convection flow past a vertical porous flat plate with variable suction," Nonlinear Analysis. Modelling and Control, vol. 11, no. 1, pp. 3-12, 2006.

[20] H. A. M. El-Arabawy, "Soret and dufour effects on natural convection flow past a vertical surface in a porous medium with variable surface temperature," Journal of Mathematics and Statistics, vol. 5, no. 3, pp. 190-198, 2009. 


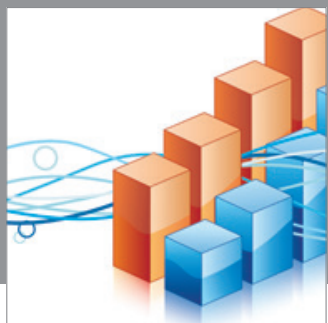

Advances in

Operations Research

mansans

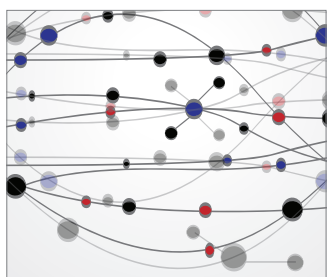

The Scientific World Journal
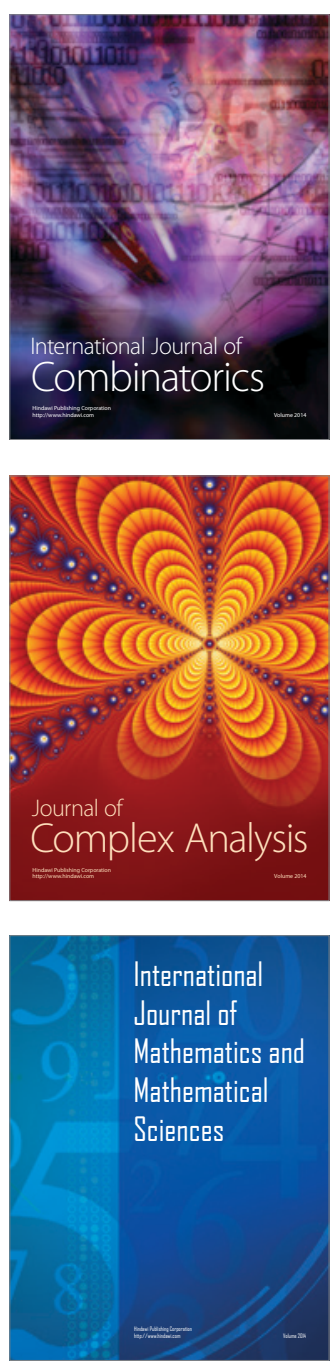
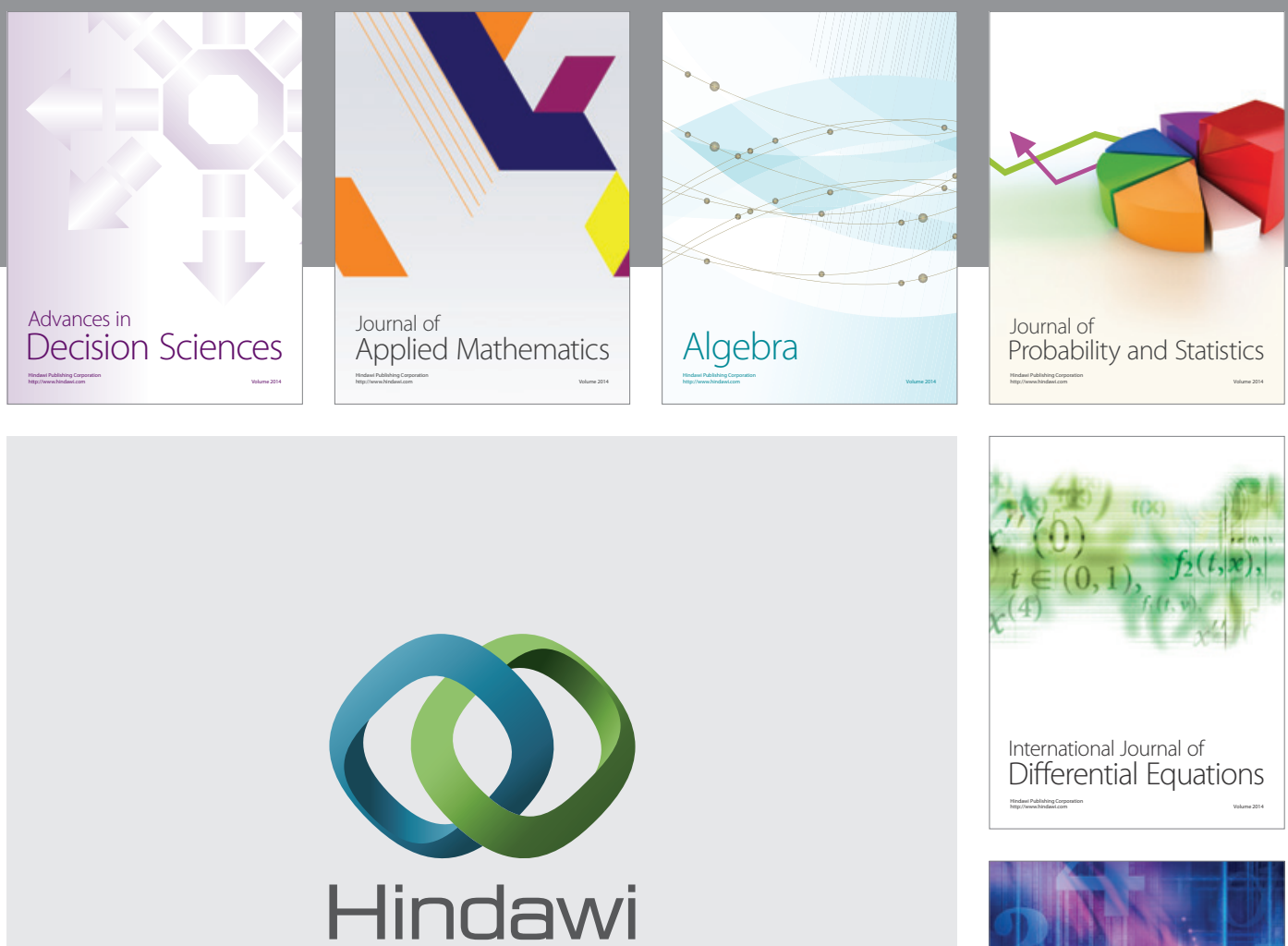

Submit your manuscripts at http://www.hindawi.com
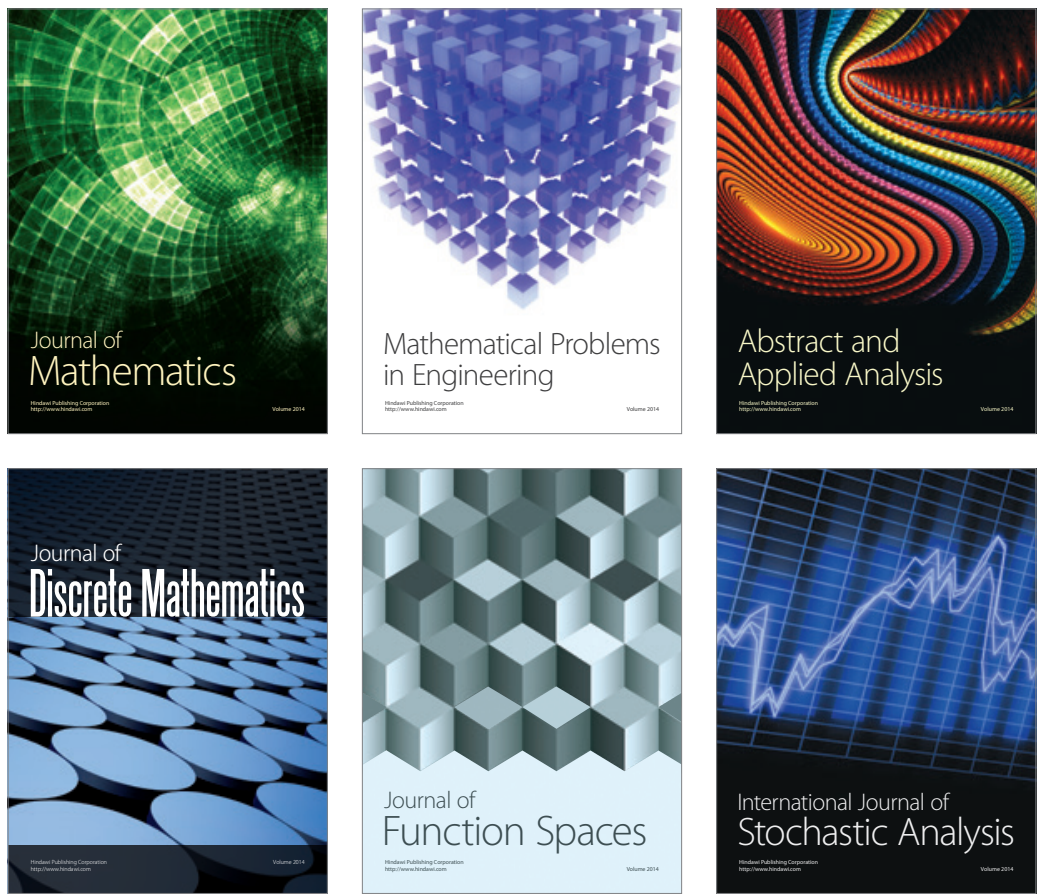

Journal of

Function Spaces

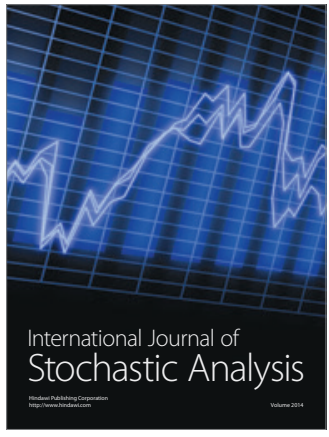

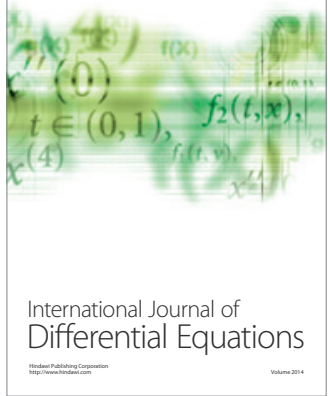
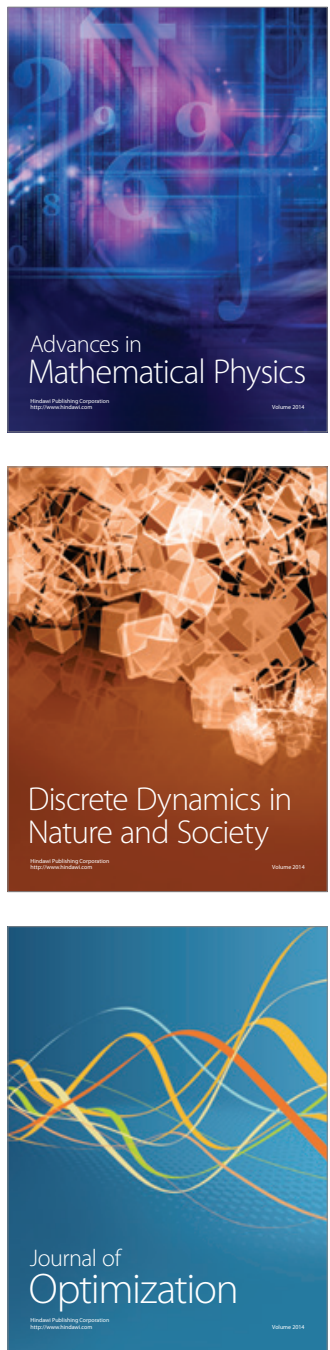